\title{
Fluctuation-dissipation relations under Lévy noises
}

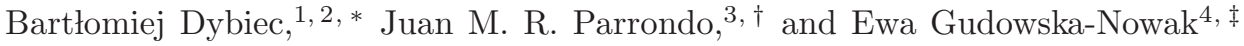 \\ ${ }^{1}$ Center for Models of Life, Niels Bohr Institute, \\ University of Copenhagen, Blegdamsvej 17, 2100 Copenhagen Ø, Denmark \\ ${ }^{2}$ Marian Smoluchowski Institute of Physics, and Mark Kac Center for Complex Systems Research, \\ Jagellonian University, ul. Reymonta 4, 30-059 Kraków, Poland \\ ${ }^{3}$ Departamento de Física Atómica, Molecular y Nuclear and GISC, \\ Universidad Complutensede Madrid, 28040-Madrid, Spain \\ ${ }^{4}$ Marian Smoluchowski Institute of Physics, and Mark Kac Center for Complex Systems Research, \\ Jagellonian University, ul. Reymonta 4, 30-059 Kraków, Poland
}

(Dated: June 13, 2018)

\begin{abstract}
For systems close to equilibrium, the relaxation properties of measurable physical quantities are described by the linear response theory and the fluctuation-dissipation theorem (FDT). Accordingly, the response or the generalized susceptibility, which is a function of the unperturbed equilibrium system, can be related to the correlation between spontaneous fluctuations of a given conjugate variable. There have been several attempts to extend the FDT far from equilibrium, introducing new terms or using effective temperatures. Recently, Prost, Joanny, and Parrondo [Phys. Rev. Lett. 103, 090601 (2009)] have shown that the FDT can be restored far from equilibrium by choosing the appropriate variables conjugate to the external perturbations. Here, we apply the generalized FDT to a system perturbed by time-dependent deterministic forces and acting under the influence of white $\alpha$-stable noises.
\end{abstract}

PACS numbers: 05.10.Gg, 05.70.Ln, 05.40.Fb.

\section{INTRODUCTION}

The fluctuation-dissipation theorem (FDT) connects correlation functions to linear response functions and constitutes a useful tool in investigations of physical properties of systems at thermodynamic equilibrium [1, 2]. By virtue of the FDT, measurable macroscopic physical quantities like specific heats, susceptibilities or compressibilities can be related to correlation functions of spontaneous fluctuations. For systems weakly displaced from equilibrium, the FDT allows one to express the linear response of physical observables to time-dependent external fields in terms of time-dependent correlation functions. Accordingly, departures from the FDT can be expected for far-from equilibrium situations and have been demonstrated in various aging, glassy and biological media $[3-6]$.

On the other hand, the wealth of theoretical, experimental and numerical research indicate that the FDT is a special case of more general fluctuation relations that remain valid also in a specific class of nonequilibrium systems [6-14]. Following a former generalization of FDT 15 based on the identity derived by Hatano and Sasa 16], we discuss here an extension of the fluctuation theorem to stochastic models obeying Markovian dynamics and driven by white $\alpha$-stable noises. We apply the generalized fluctuation-response theorem to this case and analyze the regime in which linear response theory becomes

\footnotetext{
* bartek@th.if.uj.edu.pl

$\dagger$ parrondo@fis.es

$\ddagger$ gudowska@th.if.uj.edu.pl
}

invalidated. We illustrate our results with the simple example of an oscillator coupled to a non-equilibrium bath whose action is represented by a Lévy white noise.

Let us review the FDT introduced in [15] and extended to arbitrary observables in [8]. The theorem applies to any Markov process $x(t)$ whose dynamics depends on a set of parameters $\vec{\lambda}$. We study the linear response of the system to perturbations $\vec{\lambda}(t)=\vec{\lambda}_{0}+\delta \vec{\lambda}(t)$ around a reference state, $\rho_{\mathrm{ss}}\left(x ; \vec{\lambda}_{0}\right)$ which is the stationary state corresponding to constant parameters $\vec{\lambda}_{0}$. Given an arbitrary observable $A(x)$, the response can be written as

$$
\langle A(t)\rangle-\langle A\rangle_{0} \simeq \int_{0}^{t} \chi_{A, \gamma}\left(t-t^{\prime}\right) \delta \lambda_{\gamma}\left(t^{\prime}\right) d t^{\prime},
$$

where $A(t) \equiv A(x(t))$ and the brackets $\langle\ldots\rangle_{0}$ indicate an average over the reference state $\rho_{\mathrm{ss}}\left(x ; \vec{\lambda}_{0}\right)$; summation over the repeated index $\gamma$ is assumed and $\chi_{A, \gamma}$ is the time-dependent susceptibility of variable $A$ with respect to variations of $\lambda_{\gamma}$ (i.e. perturbations of the $\gamma$ component of $\vec{\lambda}$ ). The FDT relates this susceptibility to correlations measured in the reference unperturbed state

$$
\chi_{A, \gamma}\left(t-t^{\prime}\right)=\frac{d}{d t}\left\langle A(t) X_{\gamma}\left(t^{\prime}\right)\right\rangle_{0},
$$

where $X_{\gamma}(x)$ is the variable conjugate to the perturbation $\lambda_{\gamma}$ which is defined as

$$
X_{\gamma}(x)=-\left.\frac{\partial \ln \rho_{\mathrm{ss}}(x ; \vec{\lambda})}{\partial \lambda_{\gamma}}\right|_{\vec{\lambda}=\vec{\lambda}_{0}} .
$$

In this definition $\rho_{\mathrm{ss}}(x ; \vec{\lambda})$ is the stationary probability distribution function (PDF) for constant values of the parameters $\vec{\lambda}$. 
If the reference state is the Gibbs equilibrium state corresponding to a temperature $k T=\beta^{-1}$ and a Hamiltonian $\mathcal{H}(x ; \vec{\lambda})$, the stationary PDF $\rho_{\mathrm{sS}}(x ; \vec{\lambda})$ assumes the form $\rho_{\mathrm{ss}}(x ; \vec{\lambda})=\exp [-\beta \mathcal{H}(x ; \vec{\lambda})] / Z(\beta, \vec{\lambda})$ and the conjugate variable reads

$$
X_{\gamma}(x)=\left.\frac{1}{k T} \frac{\partial[\mathcal{H}(x ; \vec{\lambda})-F(\beta, \vec{\lambda})]}{\partial \lambda_{\gamma}}\right|_{\vec{\lambda}_{=} \vec{\lambda}_{0}},
$$

where $F=-k T \ln Z$ stands for the free energy. Accordingly, $X_{\gamma}$ can be interpreted as the fluctuation of the quantity $\left.\frac{\partial \mathcal{H}\left(x ; \vec{\lambda}_{0}\right)}{\partial \lambda_{\gamma}} \equiv \frac{\partial \mathcal{H}(x ; \vec{\lambda})}{\partial \lambda_{\gamma}}\right|_{\vec{\lambda}=\vec{\lambda}_{0}}:$

$$
X_{\gamma}(x)=\frac{1}{k T}\left[\frac{\partial \mathcal{H}\left(x ; \vec{\lambda}_{0}\right)}{\partial \lambda_{\gamma}}-\left\langle\frac{\partial \mathcal{H}\left(x ; \vec{\lambda}_{0}\right)}{\partial \lambda_{\gamma}}\right\rangle_{0}\right] .
$$

For instance, if $\lambda_{\gamma}$ is a force coupled to a coordinate $x_{\gamma}$, i.e., if the control parameter appears in the Hamiltonian as $-\lambda_{\gamma} x_{\gamma}$, then the conjugate variable $X_{\gamma}=-\left(x_{\gamma}-\right.$ $\left.\left\langle x_{\gamma}\right\rangle\right) /(k T)$ represents fluctuations of $x_{\gamma}$.

On the other hand, if the reference state $\rho_{\mathrm{SS}}\left(x ; \vec{\lambda}_{0}\right)$ is not an equilibrium state, the conjugate variables defined by Eq. (3) do not have any straightforward physical interpretation [8, 15]. In this Letter, we apply the generalized FDT to a system obeying non-equilibrium Markovian dynamics driven by Lévy white noise. The system of this type may be conceived as a generalization of Brownian motion: the particle undergoing Lévy superdiffusion is performing motion with random jumps and step lengths following a power-law distribution. As a result, the width of the distribution of particles grows superlinearly with time [17, 18] signaling anomalous dynamics. A characteristic feature of Lévy flights is the dominance of rare but large jumps. This type of anomalous transport has been found ubiquitous in nature [17, 19] and serves as a suitable model for various physical phenomena like atmospheric turbulence [20], transport in turbulent plasmas 21], activation kinetics in non-equilibrium baths [22], transport in fractured materials 23], epidemic spreading [24], dispersal of banknotes 25] or light scattering in heterogeneous dielectric media [26].

\section{LINEAR SYSTEM DRIVEN BY LÉVY WHITE NOISES}

We proceed to discuss response properties of an overdamped Lévy-Brownian particle moving in a parabolic potential that is subject to a deterministic timedependent force field $f(t)$ and a white Lévy noise $\zeta(t)$ resulting from the fluctuating environment. The corresponding Langevin equation reads

$$
\left\{\begin{array}{l}
\dot{x}(t)=-a x+f(t)+\zeta(t) \\
x(0)=x_{0}
\end{array}\right.
$$

The white Lévy noise $\zeta(t)$ is defined as the time derivative of a stationary Lévy process [17, 27], i.e., the integral over time

$$
L_{\alpha, \beta}(t) \equiv \int_{0}^{t} \zeta(s) d s=z(t)
$$

represents a stochastic process with independent increments whose probability density $p_{\alpha, \beta}(z, t)$ is a stable Lévy distribution. Consequently, the Fourier transform of the probability density (characteristic function) $\varphi(k, t)=\int_{-\infty}^{\infty} e^{i k z(t)} p_{\alpha, \beta}\left(z, t ; \sigma_{0}, \mu\right) d z$ reads

$$
\varphi(k, t)=\exp \left[i k \mu_{0} t-\sigma_{0}^{\alpha}|k|^{\alpha} t\left(1-i \beta \operatorname{sign}(k) \tan \frac{\pi \alpha}{2}\right)\right]
$$

for $\alpha \neq 1$ and

$$
\varphi(k, t)=\exp \left[i k \mu_{0} t-\sigma_{0}|k| t\left(1+i \beta \frac{2}{\pi} \operatorname{sign}(k) \ln |k|\right)\right]
$$

for $\alpha=1[28]$. Here $\alpha \in(0,2]$ is the Lévy (stability) in$\operatorname{dex}, \beta \in[-1,1]$ is the skewness parameter (for $\beta=0$ the distributions are symmetric), $\sigma_{0}>0$ represents the noise intensity, and $\mu_{0} \in \mathbb{R}$ is a location (shift) parameter. The Gaussian distribution is a special case of symmetric stable distribution with $\alpha=2$ and $\beta=0$. In this case $\mu_{0} t$ is the mean of the Gaussian random variable $z(t)$ and $\sigma_{0}^{2} t$ is its variance. For $\alpha<2$, stable probability densities exhibit long tails and divergent moments: the asymptotic (large $z$ ) behavior of the corresponding PDF is then characterized by a power-law $p_{\alpha, \beta}\left(z, t ; \sigma_{0}, \mu\right) \propto|z|^{-(1+\alpha)}$. Under those circumstances, equation ([6) is associated with the space-fractional Fokker-Planck-Smoluchowski equation (FFPE) 29 31]:

$$
\begin{aligned}
& \frac{\partial p(x, t)}{\partial t}=-\frac{\partial}{\partial x}\left[\mu_{0}-a x+f(t)\right] p(x, t) \\
& +\sigma_{0}^{\alpha} \frac{\partial^{\alpha}}{\partial|x|^{\alpha}} p(x, t)+\sigma_{0}^{\alpha} \beta \tan \frac{\pi \alpha}{2} \frac{\partial}{\partial x} \frac{\partial^{\alpha-1}}{\partial|x|^{\alpha-1}} p(x, t) .
\end{aligned}
$$

Here, the fractional (Riesz-Weyl) derivative is defined by its Fourier transform $\mathcal{F}\left[\frac{\partial^{\alpha}}{\partial|x|^{\alpha}} f(x)\right]=-|k|^{\alpha} \mathcal{F}[f(x)]$ [32, 33]. Accordingly, Eq. (10) has the following Fourier representation

$$
\begin{aligned}
\frac{\partial \hat{p}(k, t)}{\partial t}= & -a k \frac{\partial}{\partial k} \hat{p}(k, t)+i k\left[\mu_{0}+f(t)\right] \hat{p}(k, t) \\
& -\sigma_{0}^{\alpha}|k|^{\alpha}\left[1-i \beta \operatorname{sign}(k) \tan \frac{\pi \alpha}{2}\right] \hat{p}(k, t),
\end{aligned}
$$

where $\hat{p}(k, t)=\mathcal{F}[p(x, t)]$. In what follows, we adhere to the analysis of strictly $\alpha$-stable random variables [27], i.e. ones for which $\mu_{0}=0$, and additionally $\beta=0$ if $\alpha=1$.

Since our original Langevin equation (6) is linear, its solution depends linearly on the stable process $L_{\alpha, \beta}(t)$. Accordingly, the probability density of the solution, $p\left(x, t \mid x_{0}, 0\right)$, has the form of an $(\alpha, \beta)$-stable Lévy distribution with time-dependent location $\mu(t)$ and scale $\sigma(t)$ 
parameters 33]. By analogy, its characteristic function is given by (cf. Eqs. (8) and (9))

$\hat{p}(k, t)=\exp \left[i k \mu(t)-\sigma^{\alpha}(t)|k|^{\alpha}\left(1-i \beta \operatorname{sign}(k) \tan \frac{\pi \alpha}{2}\right)\right]$.

We can now insert this ansatz into the Fokker-PlanckSmoluchowski equation (11). Since the derivative with respect to $k$ appears multiplied by $k$ in (11), the non analyticity of $|k|^{\alpha}$ at $k=0$ does not create any singularity in the equation. The real part of Eq. (11) yields the following evolution equation for the scale parameter $\sigma(t)$

$$
-\alpha \sigma^{\alpha-1} \dot{\sigma}=a \alpha \sigma^{\alpha}-\sigma_{0}^{\alpha}
$$

whereas the imaginary part gives

$$
\begin{aligned}
{[\dot{\mu}+a \mu-f(t)] k=} & {\left[-\alpha \sigma^{\alpha-1} \dot{\sigma}-a \alpha \sigma^{\alpha}+\sigma_{0}^{\alpha}\right] } \\
& \times \beta \tan \frac{\pi \alpha}{2}|k|^{\alpha} \operatorname{sign}(k) .
\end{aligned}
$$

The right hand side of Eq. (13) vanishes due to Eq. (12). From the left hand side one gets the evolution equation for the location parameter $\mu(t)$ :

$$
\dot{\mu}(t)=-a \mu+f(t) .
$$

The evolution equations (12) and (14) are completed with the initial conditions $\mu(0)=x_{0}$ and $\sigma(0)=0$ (we are calculating probability densities conditioned to $x(0)=$ $\left.x_{0}\right)$. The solution of these differential equations are

$$
\mu(t)=e^{-a t} x_{0}+e^{-a t} \int_{0}^{t} e^{a s} f(s) d s
$$

and

$$
\sigma(t)=\sigma_{0}\left[\frac{1}{a \alpha}\left(1-e^{-a \alpha t}\right)\right]^{1 / \alpha}
$$

where $\sigma_{0}$ is the scale parameter of the corresponding $\alpha$-stable density. For a constant force $f(t) \equiv f$, the long time asymptotics of the above equations are $\lim _{t \rightarrow \infty} \mu(t)=f / a$ and $\lim _{t \rightarrow \infty} \sigma(t)=\sigma_{0} /(a \alpha)^{1 / \alpha}$.

\section{THE CONJUGATE VARIABLE}

To determine the conjugate variable to the external force, we need the stationary distribution in position space for a constant force $f$. Despite the characteristic functions of stable distributions assume closed expressions, the corresponding PDFs have a known simple analytical form [27, 34] only in a few cases: For $\alpha=2$ and $\beta=0$ the resulting distribution is Gaussian; for $\alpha=1, \beta=0$ one gets the Cauchy distribution; finally, for $\alpha=1 / 2, \beta=1$ one gets the Lévy-Smirnoff distribution. In this section, we give explicit expressions for the conjugate variable for these three cases.
For $\alpha=2$ and $\beta=0$, the time dependent solution of the corresponding Langevin Eq. (6) is

$$
p_{2,0}\left(x, t \mid x_{0}, 0\right)=\frac{1}{\sqrt{2 \pi \sigma^{2}(t)}} \exp \left[-\frac{(x-\mu(t))^{2}}{2 \sigma^{2}(t)}\right]
$$

with $\mu(t)$ and $\sigma(t)$ given by Eqs. (15) and (16). The stationary solution $p_{\mathrm{sS}}(x)$ for a constant force $f$ is obtained by replacing $\mu(t)$ and $\sigma^{2}(t)$ by their stationary values, $f / a$ and $\sigma_{0}^{2} /(2 a)$, respectively. We then get the non-equilibrium potential

$$
\phi \equiv-\ln p_{\mathrm{ss}}(x)=-\frac{1}{2} \ln \frac{a}{\pi \sigma_{0}^{2}}+\frac{a(x-f / a)^{2}}{\sigma_{0}^{2}}
$$

and the conjugate variable can be easily derived as

$$
X_{\mathrm{G}}=\left.\frac{\partial \phi}{\partial f}\right|_{f=0}=-\frac{2 x}{\sigma_{0}^{2}}
$$

which is proportional to $x$, as expected, since the Gaussian case corresponds to a Brownian particle in equilibrium.

For $\alpha=1$ and $\beta=0$, the time dependent solution of the corresponding Langevin Eq. (6) is the Cauchy distribution

$$
p_{1,0}\left(x, t \mid x_{0}, 0\right)=\frac{\sigma(t)}{\pi} \frac{1}{[x-\mu(t)]^{2}+\sigma^{2}(t)}
$$

and the stationary solution for a constant force $f$ is obtained replacing $\mu(t)$ and $\sigma(t)$ by their stationary values, $f / a$ and $\sigma_{0} / a$, respectively. The non-equilibrium potential in this case is given by

$$
\phi=-\ln \frac{\sigma_{0}}{a \pi}+\ln \left[(x-f / a)^{2}+\left(\sigma_{0} / a\right)^{2}\right],
$$

so that the conjugate variable takes the form

$$
X_{\mathrm{C}}=-\frac{2 x}{a\left[x^{2}+\left(\sigma_{0} / a\right)^{2}\right]}
$$

which is proportional to $x$ only for small values of $x$ and becomes proportional to $1 / x$ for large $x$. This large $x$ behavior ensures the convergence of all the moments of $X_{C}$, whereas for the Cauchy case $|x|^{\nu}$ exists only if $\nu<1$, see 27]

Finally, for $\alpha=1 / 2$ and $\beta=1$ the solution to Eq. (6) is the Lévy-Smirnoff PDF

$$
p_{1 / 2,1}\left(x, t \mid x_{0}, t_{0}\right)=\sqrt{\frac{\sigma(t)}{2 \pi[x-\mu(t)]^{3}}} \exp \left[-\frac{\sigma(t)}{2(x-\mu(t))}\right]
$$

for $x>\mu(t)$ and $p_{1 / 2,1}\left(x, t \mid x_{0}, 0\right) \equiv 0$ for $x \leq \mu(t)$. The stationary values of $\mu(t)$ and $\sigma(t)$ are in this case $f / a$ and $4 \sigma_{0} / a^{2}$, respectively. Inserting these values, one can easily obtain the non-equilibrium potential $\phi=-\ln p_{\mathrm{ss}}(x)$ and the conjugate variable

$$
X_{\mathrm{L}-\mathrm{S}}=\frac{4 \sigma_{0}-3 a^{2} x}{2 a^{3} x^{2}} ; \quad x>0 .
$$




\section{SUSCEPTIBILITY AND RESPONSE}

The main objective of the current work is to compare the response of the system to external perturbation as calculated directly from the definition

$$
\langle X(t)\rangle=\int_{-\infty}^{\infty} X(x) p(x, t) d x,
$$

or, otherwise determined by the generalized susceptibility $\chi(t)=\frac{d}{d t}\langle X(t) X(0)\rangle_{0}$ within linear response theory:

$$
\langle X(t)\rangle_{\mathrm{LR}}=\int_{0}^{t} \chi(t-s) f(s) d s .
$$

We restrict our analysis to the Cauchy case, $\alpha=1, \beta=$ 0 , and denote the conjugate variable as $X \equiv X_{\mathrm{C}}$, with $X_{\mathrm{C}}$ given by Eq. (22). In this case, the time-dependent average (25) can be calculated exactly with the probability density:

$$
p(x, t)=\int_{-\infty}^{\infty} p\left(x, t \mid x_{0}, 0\right) p\left(x_{0}\right) d x_{0}
$$

where

$$
p\left(x_{0}\right)=\frac{\sigma_{0}}{a \pi} \frac{1}{x_{0}^{2}+\left(\sigma_{0} / a\right)^{2}}
$$

and $p\left(x, t \mid x_{0}, 0\right)$ is given by Eq. (20).

On the other hand, the FDT relates the susceptibility with the autocorrelation of the conjugate variables in the reference state, i.e., for $f=0$. The autocorrelation is defined as

$$
\begin{aligned}
\langle X(t) X(0)\rangle_{0}=\iint & \frac{2 x}{a\left[x^{2}+\left(\sigma_{0} / a\right)^{2}\right]} \frac{2 y}{a\left[y^{2}+\left(\sigma_{0} / a\right)^{2}\right]} \\
& \times \frac{\sigma(t)}{\pi\left[(x-\mu(t))^{2}+\sigma^{2}(t)\right]} \\
& \times \frac{\sigma_{0}}{a \pi\left[y^{2}+\left(\sigma_{0} / a\right)^{2}\right]} d x d y
\end{aligned}
$$

where $\mu(t)=e^{-a t} y$ and $\sigma(t)=\sigma_{0}\left[\left(1-e^{-a t}\right) / a\right]$. The final result is surprisingly simple:

$$
\langle X(t) X(0)\rangle_{0}=\frac{1}{2 \sigma_{0}^{2}} e^{-a t} .
$$

From the above, the generalized susceptibility can be derived by differentiation with respect to time (see Eq. (2)):

$$
\chi(t)=\frac{d}{d t}\langle X(t) X(0)\rangle_{0}=-\frac{a}{2 \sigma_{0}^{2}} e^{-a t} .
$$

In further calculations, for the sake of simplicity, it is assumed that $a=1$ and $\sigma_{0}=1$, so that $\langle X(t) X(0)\rangle=$ $\frac{1}{2} e^{-t}$ and $\chi(t)=-\frac{1}{2} e^{-t}$.

In order to test the linear response theory for our dynamic Markov system subjected to Lévy white noise, we calculate the response of the conjugate variable $X$ to two different time dependent perturbations: the sum of a
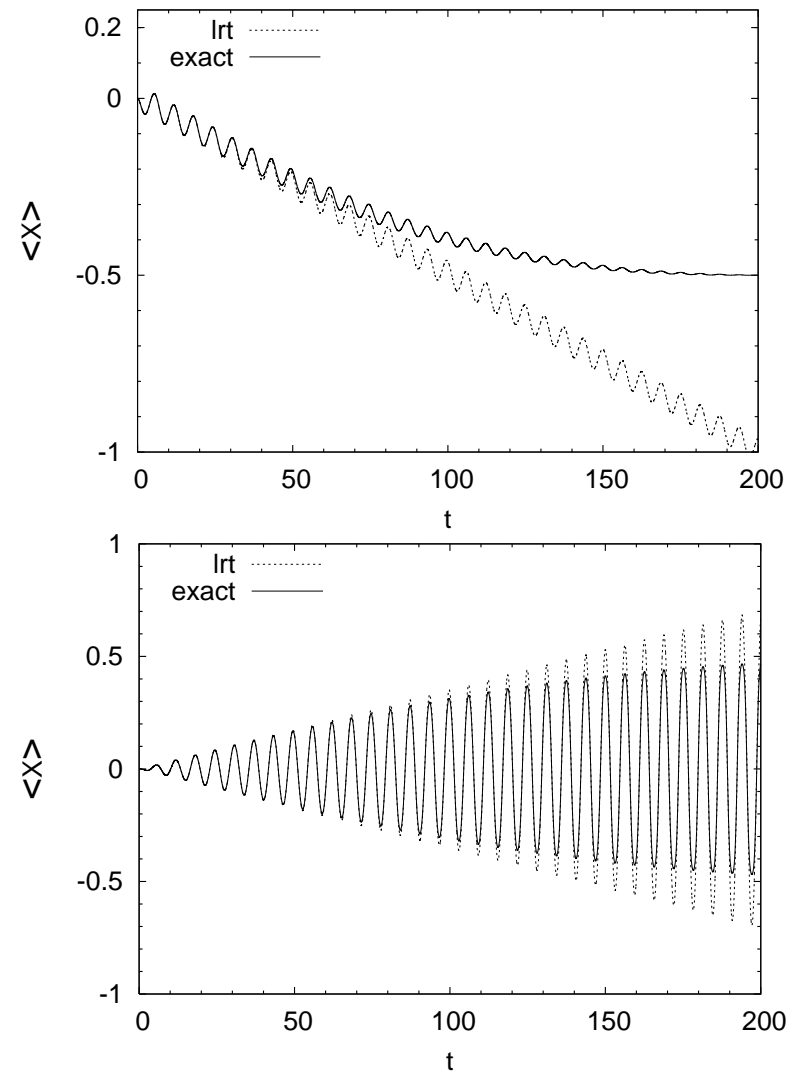

FIG. 1. Response of $\langle X(t)\rangle$ to external drivings $f_{1}(t)=$ $\sin (t) / 10+t / 100$ (upper plot) and $f_{2}(t)=t \sin (t) / 100$ (lower plot). The solid and dotted lines present an exact result (Eq. (25) and a result constructed by use of the linear response theory (Eq. 26) ), respectively.

small periodic and a linearly increasing force, $f_{1}(t)=$ $\sin (t) / 10+t / 100$; and a periodic force with increasing amplitude, $f_{2}(t)=t \sin (t) / 100$. Figure 1 displays the exact evolution of $\langle X(t)\rangle$ and the result obtained from the linear response theory. For small perturbations, (i.e. short times) in both cases, linear response theory yields an accurate estimation of the response. In the case of $f_{1}(t)$, for large times the response $\langle X(t)\rangle$ is insensitive to the sinusoidal component of the force, which is small compared with the linear part. This is due to the peculiar form of the conjugate variable $X$ given by Eq. (22). For a constant force $f$, the mean value of $X$ is

$$
\begin{aligned}
\langle X\rangle & =-\frac{\sigma_{0}}{a \pi} \int_{-\infty}^{\infty} \frac{d x}{[x-f / a]^{2}+\left(\sigma_{0} / a\right)^{2}} \frac{2 x}{a\left[x^{2}+\left(\sigma_{0} / a\right)^{2}\right]} \\
& =-\frac{2 f}{f^{2}+4 \sigma_{0}^{2}}
\end{aligned}
$$

which yields $\langle X\rangle=-0.5$ for $\sigma_{0}=1$ and $f=2$ (at $t=200, f_{1}(t) \simeq 2$ ). A similar saturation effect is not observed for the sinusoidal force $f_{2}(t)$.

We can apply the FDR to any function $A(x)$ with finite average. Due to the long tails of stable distributions, only moments $\left\langle|x|^{\nu}\right\rangle$ with $\nu<\alpha$ converge $(\nu<1$ in the 
case of Cauchy distributions) [27]. Moreover, those moments are even functions of $x$ and, for symmetry reasons, the correlation with $X$ vanishes: $\left\langle|x(t)|^{\nu} X(0)\right\rangle=0$. Consequently, the deviation of $\left\langle|x(t)|^{\nu}\right\rangle$ with respect to its reference value is non linear in the perturbation $f(t)$. On the other hand, we can obtain non-trivial results for odd fractional moments $A(x)=\operatorname{sign}[x(t)]|x(t)|^{\nu}$, whose average in the reference state vanishes $\langle A\rangle_{0}=0$. The corresponding correlation function reads

$$
\langle A(x(t)) X(0)\rangle=-\frac{\nu}{\sin [\pi \nu / 2]} e^{-t}
$$

and the generalized susceptibility is given by

$$
\chi_{A}(t)=\frac{\nu}{\sin [\pi \nu / 2]} e^{-t} .
$$

In the spirit of the former definition, see Eq. (25), the exact value of $\langle A(x(t))\rangle$ can be calculated as

$$
\begin{aligned}
\langle A(x(t))\rangle & =\int_{-\infty}^{\infty} A(x(t)) p(x, t) d x \\
& =\int_{-\infty}^{\infty} A(x(t)) p\left(x, t \mid x_{0}, 0\right) p\left(x_{0}\right) d x,
\end{aligned}
$$

where $p\left(x_{0}\right)$ and $p\left(x, t \mid x_{0}, 0\right)$ are given by Eqs. (20) and (28) respectively. Figure 2 displays the comparison of the exact evolution $\left\langle\operatorname{sign}[x(t)]|x(t)|^{1 / 2}\right\rangle$ with the linear response approximation $\int_{0}^{t} \chi_{A}(t-s) f(s) d s$. As previously, linear response theory is valid for weak perturbation up to $f \simeq 0.5$. However, for the fractional moment and the linearly increasing force (upper plot), we do not observe saturation.

\section{SUMMARY AND CONCLUSIONS}

We have shown that the generalized FDT can be applied to linear systems driven by Lévy noise. The FDT allows one to calculate the susceptibility of any observable and then the response to any small time dependent perturbation. For a noise distributed according to the Cauchy distribution, we have calculated the susceptibility of the conjugate variable $X_{\mathrm{C}}$ and the susceptibility of odd fractional moments $\left\langle\operatorname{sign}[x(t)]\left|x(t)^{\nu}\right|\right\rangle$, which have a simple exponential behavior. From these susceptibilities it is easy to get simple analytical expressions for the response of the system using Eq. (26). We have to notice that, although the exact response can be calculated analytically using Eq. (25), the corresponding integrals are cumbersome and can be only solved numerically in the simplest cases. Therefore, the generalized FDT is shown to be a useful analytical tool to deal with these type of systems.

It is still not obvious whether the conjugate variables that we have calculated for the Cauchy and LévySmirnoff noises have any physical meaning, besides the one provided by the generalized FDT itself. The generalized FDT shows that these conjugate variables represent
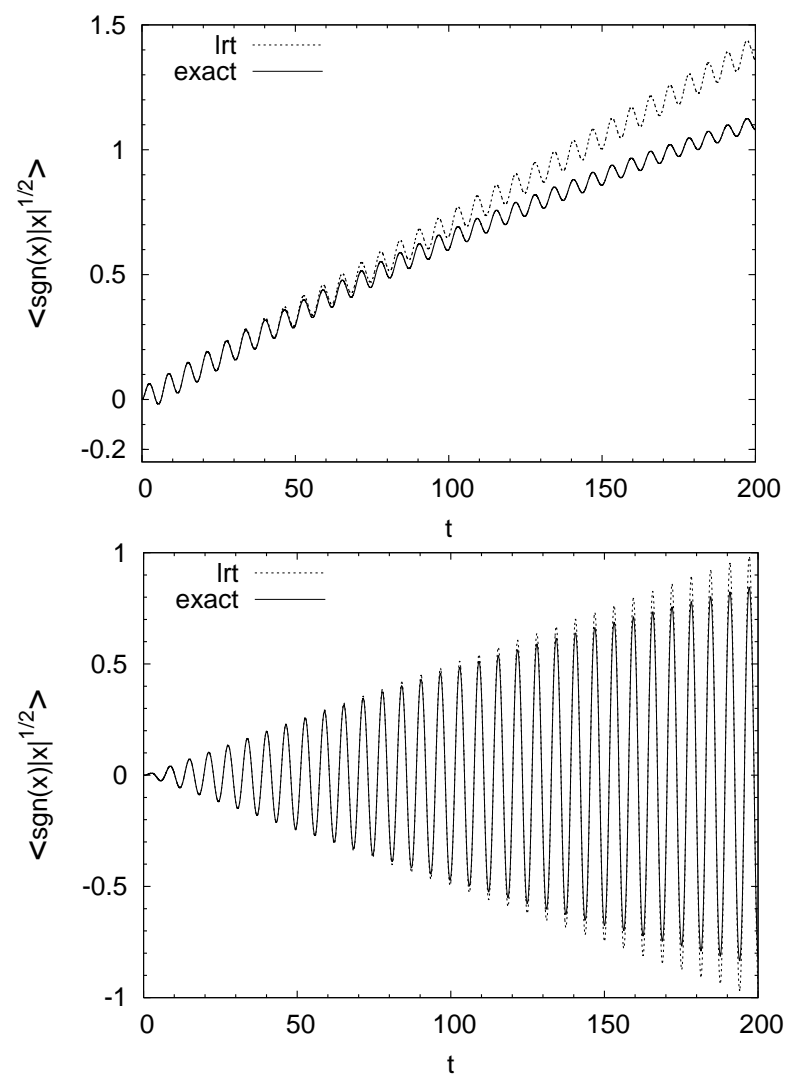

FIG. 2. Response of the $\left\langle\operatorname{sign}[x(t)]|x(t)|^{1 / 2}\right\rangle$ to $f(t)=$ $\sin (t) / 10+t / 100$ (upper plot) and $f(t)=t \sin (t) / 100$ (lower plot). The solid line presents exact results, see Eq. (33), while the dotted line results constructed by use of the linear response theory, see Eqs. (26) and (31).

the change in the probability distribution of the system under the perturbation. In equilibrium, this change is also related with the energy that the system absorbs from the perturbation. On the other hand, for non-equilibrium systems, the lack of conserved quantities prevents such an interpretation. For instance, in the case of the harmonic oscillator driven by a Cauchy-Lévy noise, $\langle x\rangle$ and higher moments diverge (cf. [35]). Consequently, both the potential energy of the system in the harmonic potential and the work done by the external force $f(t)$ also diverge. The system is plagued by divergent quantities. However, the conjugate variable $X_{\mathrm{C}}$ given by Eq. (22) has finite moments and still captures the dynamical response of the Lévy particle. Summarizing, although systems driven by $\alpha$-stable noises might significantly differ from their Brownian (equilibrium) counterparts [36 38] due to their heavy tail asymptotics, we have shown that in such farfrom-equilibrium situations some concepts from weakly perturbed equilibrium systems can be still used.

One of the drawbacks of the generalized FDT derived in [15] is the difficulty to find the conjugate variable, since it requires the knowledge of the stationary state. We have been able to find this stationary state for a linear system. 
An interesting open question is whether this state, or some slight modification, can still be used to calculate susceptibilities in the presence of weak non-linearities.

\section{ACKNOWLEDGMENTS}

We are grateful to Jordan Horowitz for reading the manuscript and providing various useful suggestions.
The authors acknowledge the support by the European Science Foundation (EFS) through Exploring Physics of Small Devices (EPSD) program. JMRP also acknowledges financial support from grants MOSAICO (Spanish Government) and MODELICO (Comunidad de Madrid). BD acknowledges the Danish National Research Foundation for financial support through the Center for Models of Life (CMOL).
[1] R. Kubo, Rep. Prog. Phys. 29, 255 (1966).

[2] M. Toda, R. Kubo, and N. Hashitsume, Statistical physics: Nonequilibrium statistical mechanics (Springer Verlag, Berlin, 1991).

[3] K. Hayashi and M. Takano, Biophys. J. 93, 895 (2007).

[4] A. Crisanti and F. Ritort, J. Phys. A: Math. Gen. 36, R181 (2003).

[5] P. Calabrese and A. Gambassi, J. Phys. A: Math. Gen. 38, R133 (2005).

[6] U. M. B. Marconi, A. Puglisi, L. Rondoni, and A. Vulpiani, Phys. Rep. 461, 111 (2008).

[7] R. Chetrite, G. Falkovich, and K. Gawedzki, J. Stat. Mech. P08005 (2008).

[8] U. Seifert and T. Speck, Europhys. Lett. 89, 1007 (2010).

[9] H. Touchette and E. G. D. Cohen, Phys. Rev. E 80, 011114 (2009).

[10] H. Touchette and E. G. D. Cohen, Phys. Rev. E 76, 020101 (2007).

[11] A. V. Chechkin and R. Klages, J. Stat. Mech. L03002 (2009).

[12] A. Dubkov, Chem. Phys. 375, 364 (2010).

[13] P. Allegrini et al., Phys. Rev. Lett. 103, 030602 (2009).

[14] W. Ebeling and I. M. Sokolov, Statistical thermodynamics and stochastic theory of nonequilibrium systems (World Scientific Publishing, Singapore, 2005).

[15] J. Prost, J.-F. Joanny, and J. M. R. Parrondo, Phys. Rev. Lett. 103, 090601 (2009).

[16] T. Hatano and S. Sasa, Phys. Rev. Lett. 86, 3463 (2001).

[17] A. A. Dubkov, B. Spagnolo, and V. V. Uchaikin, Int. J. Bifurcation Chaos. Appl. Sci. Eng. 18, 2649 (2008).

[18] B. Dybiec and E. Gudowska-Nowak, Phys. Rev. E 80, 061122 (2009).

[19] Lévy processes: Theory and applications, edited by O. E. Barndorff-Nielsen, T. Mikosch, and S. I. Resnick (Birkhäuser, Boston, 2001).

[20] M. F. Shlesinger, J. Klafter, and B. J. West, Physica A 140, 212 (1986).
[21] R. Sánchez et al., Phys. Rev. Lett. 101, 205002 (2008).

[22] B. Dybiec and E. Gudowska-Nowak, Phys. Rev. E 69, 016105 (2004).

[23] C.-C. Lee, H.-C. Lee, H.-F. Yeh, and H.-I. Lin, Environ. Earth Sci. 63, 1199 (2010).

[24] B. Dybiec, A. Kleczkowski, and C. A. Gilligan, J. R. Soc. Interface 6, 941 (2009).

[25] D. Brockmann, L. Hufnagel, and T. Geisel, Nature (London) 439, 462 (2006).

[26] P. Barthelemy, J. Bertolotti, and D. Wiersma, Nature (London) 453, 495 (2008).

[27] A. Janicki and A. Weron, Simulation and chaotic behavior of $\alpha$-stable stochastic processes (Marcel Dekker, New York, 1994).

[28] W. Feller, An introduction to probability theory and its applications (John Wiley, New York, 1968).

[29] R. Metzler, E. Barkai, and J. Klafter, Europhys. Lett. 46, 431 (1999).

[30] V. V. Yanovsky, A. V. Chechkin, D. Schertzer, and A. V. Tur, Physica A 282, 13 (2000).

[31] D. Schertzer et al., J. Math. Phys. 42, 200 (2001).

[32] I. Podlubny, Fractional differential equations (Academic Press, San Diego, 1999).

[33] S. Jespersen, R. Metzler, and H. C. Fogedby, Phys. Rev. E 59, 2736 (1999).

[34] K. A. Penson and K. Górska, Phys. Rev. Lett. 105, 210604 (2010).

[35] With a more general definition of integrals, i.e. by using a "principal value" integral, the first moment of a Cauchy distribution can be evaluated as $\langle x\rangle=$ $\lim _{a \rightarrow \infty} \frac{1}{\pi} \int_{-a}^{a} \frac{x}{1+x^{2}} d x$ and is equal zero

[36] A. V. Chechkin et al., Chem. Phys. 284, 233 (2002).

[37] B. Dybiec and E. Gudowska-Nowak, J. Stat. Mech. P05004 (2009).

[38] B. Dybiec, A. V. Chechkin, and I. M. Sokolov, J. Stat. Mech. P07008 (2010). 